\title{
Structural and Magnetic Properties of Mn-Based Diluted Magnetic Semiconductors and Alloys
}

\author{
A. Alsaad \\ Department of Physical Sciences, Jordan University of Science \& Technology, P. O. Box 3030, Irbid 221100, Jordan \\ Correspondence should be addressed to A. Alsaad, alsaad11@just.edu.jo
}

Received 21 June 2009; Accepted 4 August 2009

Recommended by Faustino Aguilera-Granja

\begin{abstract}
Direct supercell approach calculations of the magnetic exchange interactions in Mn-doped ScN was carried out in the local spin density approximation by using the muffin-tin-orbital Green's function method. We found that magnetic interactions are long range interactions and affected by the randomness, band gap corrections, and carrier concentrations. Using total energy minimization approach we found that the global energy minimum of $\mathrm{MnN}$ is obtained for zinc-blende structure. If the compound is compressed by $6 \%$, the energy minimum corresponds to the rock-salt structure in disagreement with the experimentally observed tetragonal distorted rock-salt structure, known as $\theta$-phase. An isostructural phase transition for $\mathrm{Mn}_{\mathrm{x}} \mathrm{Sc}_{1-\mathrm{x}} \mathrm{N}$ alloys from $\mathrm{MnN} \theta$-phase to $h$-ScN phase was found to occur at a hydrostatic pressure of $18 \mathrm{GPa}$. We predict above room temperature $T_{c}$ for Mn concentrations of about $10 \%$ in $\mathrm{ScN}$ : Mn system.
\end{abstract}

Copyright ( 2009 A. Alsaad. This is an open access article distributed under the Creative Commons Attribution License, which permits unrestricted use, distribution, and reproduction in any medium, provided the original work is properly cited.

\section{Introduction}

The undergoing intensive research and the current industrial and technological interests in the field of spintronics stimulate the search for high-Curie-temperature diluted magnetic semiconductors (DMSs). The typical zinc blende III-V semiconductors doped with magnetic impurities suffer from low solubility manifested in the formation of secondary phases. Clearly, there is an interest in finding an alternative semiconductor host and magnetic dopants like Mn with $T_{c}$ above room temperature. One limitation of the traditional semiconductors tried up to now is the small solubility of magnetic dopants like $\mathrm{Mn}$ in them. To conquer this problem we, alternatively, propose the less known rock-salt structure ScN semiconductors, because it shares a common crystal structure with magnetic (or antiferromagnetic) transition metal nitrides [1]. The growth of mixed $\mathrm{Mn}_{\mathrm{x}} \mathrm{Sc}_{1-\mathrm{x}} \mathrm{N}$ alloys using molecular beam epitaxial growth technique has been reported [2]. In our study, the exchange magnetic interactions were investigated by fitting the total energies of nearestneighbor pairs of parallel and antiparallel $\mathrm{Mn}$ spins to a Heisenberg Hamiltonian. The interactions with near and distant cells were considered. The nearest neighbor exchange interactions can be written as

$$
H=\sum_{\langle i j\rangle} J_{i j} \mathbf{S}_{i} \cdot \mathbf{S}_{j} .
$$

We found that exchange magnetic interactions are long range and drastically affected by the randomness of the cell as was observed in other DMSs $[3,4]$. The search for magnetic semiconductors with a curie temperature $T_{c}$ above room temperature (RT) is currently one of the major issues in semiconductor spintronics [5]. For $\mathrm{MnN}$, three different phases, including RS, ZB, and WZ are considered. Both ferromagnetic (FM) and antiferromagnetic (AFM) states are calculated for each compound in the three phases. The binary $\mathrm{ScN}$ is an RS semiconductor with in-plane lattice constant ranging between 4.50 and $4.503 \AA$, indirect band gap from $\Gamma-\mathrm{X}$ of approximately $1 \mathrm{eV}$, and a direct transition at $\mathrm{X}$ point of 2.1-2.4 eV [6]. In addition, experimental studies and theoretical calculations have confirmed that $\mathrm{ScN}$ stabilizes in a fivefold coordinated structure called $h$-ScN [7]. On the other hand, $\mathrm{Mn}$ is an early transition metal and has slightly tetragonal distorted rock-salt, known as $\theta$-phase [8]. Thus, it is of great interest to combine $\mathrm{MnN}$ with $\mathrm{ScN}$ to yield Mn-doped ScN DMSs and ferromagnetic $\mathrm{Mn}_{\mathrm{x}} \mathrm{Sc}_{1-\mathrm{x}} \mathrm{N}$ alloys. 
Within mean field theory, the Curie temperature has been estimated to be above $400 \mathrm{~K}$ for $3 \% \mathrm{Mn}$.

\section{Methodology}

First-principle calculations within the framework of the density functional theory $[9,10]$ and spin local density approximation (SLDA) $[11,12]$ are performed. We employed the full-potential linearized-augmented plane-wave (FPLAPW) method as well as linearized muffin-tin orbital (LMTO) both in the atomic sphere approximation (ASA) and in a full potential (FP) as used in the Wien2k code [13] and WIEN97 code [14] applied to a 2-atom unit cell because they allow a shift in the diagonal elements of the Hamiltonian in a straightforward manner. Therefore, they allow us to correct the LDA underestimate of the band gap. The muffin-tin radius adopted were $1.75 \mathrm{a}$. u. for $\mathrm{Mn}$ and 1.44 a. u. for $\mathrm{N}$ in $\mathrm{MnN}$. The total energy and eigen values were converged to $10^{-5} \mathrm{eV}$ and $10^{-4} \mathrm{eV}$, respectively. The calculations were performed with spin-polarized potentials in order to analyze the ferromagnetic phases of MnN. A $6 \times 6 \times 6 k$-point mesh was used to ensure the convergence of Brillouin zone integrations. To study the exchange magnetic interactions as a function of Mn-Mn pairs and the effects of carrier concentration and doping on Curie temperature, we used a 64 supercell with cutoff energy of 25 Ry to ensure full convergence.

\section{Results}

3.1. Magnetic Stability, Magnetic Moment, and the Phase Transition in Mn-Doped ScN DMS and $M_{\mathbf{n}} S \mathbf{c}_{1-\mathbf{x}} N$ Alloys. Our total energy calculations of $\mathrm{MnN}$ as a function of lattice constant $a$ in RS, WZ, and ZB in Ferromagnetic (FM) and antiferromagnetic (AFM) states indicate that the global energy minimum is obtained for $\mathrm{ZB}$ structure. If the compound is compressed by $6 \%$, the energy minimum corresponds to the RS structure. This result disagrees with a slightly tetragonal distorted RS structure, known as $\theta$ phase experimentally found for $\mathrm{MnN}$ binary. From the curvature of total energy as a function of $a$ for the AFM state, we deduce a bulk modulus $\mathrm{B}=259 \mathrm{GPa}$ (see Table 1 ). Next, we consider the different magnetic configurations of $\mathrm{MnN}$. Our results are summarized in Table 2. The energy difference between FM and AFM is $\Delta \mathrm{E}=0.031 \mathrm{eV} /$ atom. Previous analysis of the partial density of states has concluded that majority states are filled with three electrons and lead to localized $S=3 / 2$ spins [1]. This is consistent with our value for the magnetic moment of $\mathrm{MnN}$ (see Table 2). Having confirmed that $\mathrm{ScN}$ : Mn can behave as a DMS, we turn our attention to study the structural properties of $\mathrm{Mn}_{\mathrm{x}} \mathrm{Sc}_{1-\mathrm{x}} \mathrm{N}$ alloys. Previously, we found that $\mathrm{ScN} / \mathrm{GaN}$ and $\mathrm{ScN} / \mathrm{InN}$ superlattices can exhibit isostructural phase transitions (IPTs) at certain values of hydrostatic pressure (see [15] and references therein). We found that IPTs were associated with anomalies optical and piezoelectric properties. In this work, we examine the possibility of IPTs in $\mathrm{Mn}_{\mathrm{x}} \mathrm{Sc}_{1-\mathrm{x}} \mathrm{N}$ alloys. Figure 1 shows the lattice constant $a$ of the $\mathrm{Mn}_{\mathrm{x}} \mathrm{Sc}_{1-\mathrm{x}} \mathrm{N}$ alloys
TABLE 1: Lattice parameters relaxation results for antiferromagnetic (AFM) MnN.

\begin{tabular}{lcc}
\hline Property & Theory & Experiment $[2]$ \\
\hline$a(\AA)$ & 4.15 & 4.22 \\
Axial ratio $c / a$ & 0.982 & 0.98 \\
$B(\mathrm{GPa})$ & 259 & \\
\hline
\end{tabular}

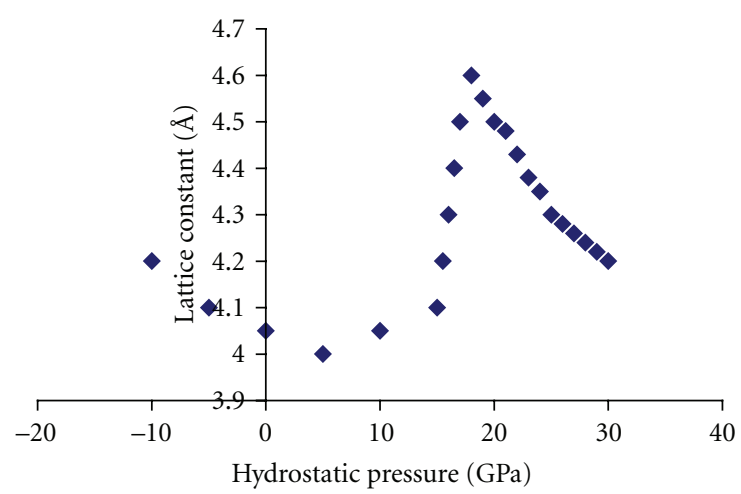

FIgure 1: Lattice constant of $\mathrm{Mn}_{\mathrm{x}} \mathrm{Sc}_{1-\mathrm{x}} \mathrm{N}$ alloys as a function of the hydrostatic pressure. An isostructural phase transition occurs at a critical pressure of $18 \mathrm{GPa}$.

as a function of the hydrostatic pressure. We started with the theoretical lattice constant of $\mathrm{ZB} \mathrm{MnN}$ and experimental lattice constant of $\theta-\mathrm{MnN}$, and we studied the variation of lattice constant with hydrostatic pressure. Figure 1 shows a remarkable phenomenon: from 5 to $18 \mathrm{GPa}$ the lattice parameter increases by $15 \%$ indicating drastic continuous structural transformations in this pressure range. Thus, anomalous optical, piezoelectric, and magnetic properties are expected. It also indicates that this alloy undergoes a sharp prerssure-induced isostructural phase transition at a critical hydrostatic pressure of $18 \mathrm{GPa}$ from the tetragonal distorted RS $\theta$-phase into the fivefold coordinated $h$-ScN. Since the synthesis of $\mathrm{Mn}_{\mathrm{x}} \mathrm{Sc}_{1-\mathrm{x}} \mathrm{N}$ alloys has been achieved for a wide range of $\mathrm{Mn}$ concentrations, we would suggest that concern experimental groups should study temperature dependence of magnetization, resistivity, and spceific heat of $\mathrm{Mn}_{\mathrm{x}} \mathrm{Sc}_{1-\mathrm{x}} \mathrm{N}$ alloys as a function of hydrostatic pressure.

\subsection{Exchange Magnetic Interactions, Critical Temperatures,} and the Effects of Carrier Concentration. We next focus on Mn-doped ScN DMS. Figure 2 plots the nearest neighbor $\mathrm{Mn}-\mathrm{Mn}$ exchange interactions as a function of the distance in $\mathrm{ScN}$ : Mn. We found that FM configurations have lower energy than AFM state. This ferromagnetic coupling is somehow surprising when keeping in mind that the coupling in $\mathrm{MnN}$ is antiferromagnetic. We explain this by the fact that the larger Mn-Mn distance will lead to less overlap between the $\mathrm{Mnt}_{2}$ orbitals, which favors ferromagnetic coupling, and also the nearest neighbor exchange interactions in $\mathrm{ScN}: \mathrm{Mn}$ are much less than in the MnN binary. In order to account for the effect of randomness, we construct relatively large supercell consists of 128 atoms. Our (SLDA) results indicate that exchange magnetic interactions follow 
TABLE 2: Lattice constant and magnetic moment of MnN in different phases for both the FM and AFM states.

\begin{tabular}{lcccc}
\hline Compound & Structure & Magnetic ordering & $a(\AA)$ & Magnetic moment $\mu\left(\mu_{\mathrm{B}}\right)$ \\
\hline $\mathrm{MnN}$ & Rock-salt & FM & 4.07 & 2.52 \\
& Wurtzite & AFM & 4.38 & 0.01 \\
& Zinc-blende & AFM & 4.30 & 0.00 \\
\hline
\end{tabular}

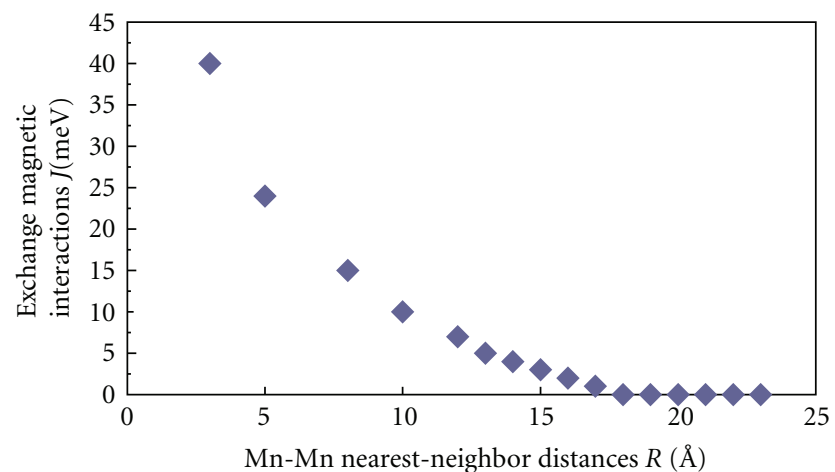

FIgURE 2: The exchange magnetic interaction $J_{i j}$ as a function of Mn-Mn nearest-neighbor distances $R_{i j}$ for the $\mathrm{ScN}: \mathrm{Mn}$ diluted magnetic semiconductor.

Ruderman-Kittel-Kasuya-Yosida (RKKY) [16, 17]. Figure 2 also indicates that strong second nearest neighbor interactions are suppressed by large exchange magnetic interactions that can be correlated with the critical temperature $T_{c}$ by averaging $J_{i j}$ over the various atoms in the cell. A good estimate for $T_{c}$ can be obtained using a mean-field approximation (MF) and even a better estimate of $T_{c}$ is obtained using the cluster variation method (CVM). For Mn concentrations up to $30 \%$, the exchange interactions of up to four nearest-neighbors are significant. The MF and CVM Curie temperatures $T_{c}^{\mathrm{MF}}$ and $T_{c}^{\mathrm{CVM}}$ as functions of Mn concentration for Mn-doped ScN are shown in Figure 3. It indicates that $T_{c}$ increases with Mn concentrations and saturates above $\mathrm{Mn}$ concentration of $28 \%$ at a maximum $T_{c}$ of $670 \mathrm{~K} . T_{c}$ above room temperatures is predicted for Mn concentration larger than $12 \%$. Thus, ferromagnetic behavior above room temperature is possible in Mn-doped $\mathrm{ScN}$. It should be noted that for small concentration of Mn (6.25\%) (a cell of 64 atoms) with Mn located only at first nearest neighbors with no second nearest neighbor pair had $T_{c}^{\mathrm{CVM}}=240 \mathrm{~K}$ and $T_{c}^{\mathrm{MF}}=306 \mathrm{~K}$. However, large supercells (128 atoms) with all Mn are located at second nearest neighbors with no $\mathrm{Mn}$ on first nearest neighbors exhibit lower values of $T_{c}^{\mathrm{CVM}}=165 \mathrm{~K}$ and $T_{c}^{\mathrm{MF}}=210 \mathrm{~K}$, respectively. Next, we consider the effects of defects affecting the carrier concentration. We can account for the effect of additional doping by simply shifting the Fermi level. Figure 4 shows $T_{c}^{\mathrm{MF}}$ as a function of electron concentration. We consider both $n$-type and $p$-type doping. Negative electron concentrations correspond to $p$-type doping, and positive values correspond to $n$-type doping. The $T_{c}^{\mathrm{MF}}$ increases and remains high up to $2 \times 10^{20} \mathrm{~cm}^{-3}$ but then drops to about zero for about $2 \times 10^{20} \mathrm{~cm}^{-3}$. Such electron concentrations

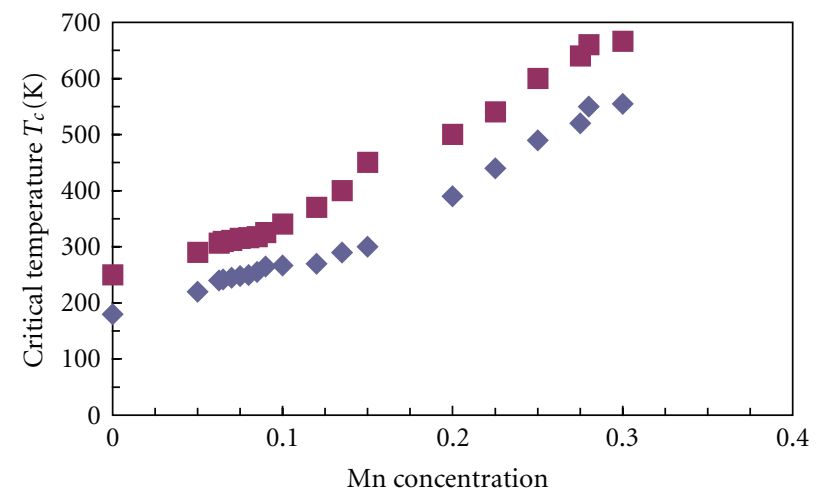

Figure 3: The critical Curie temperature versus Mn concentration for $\mathrm{Mn}$-doped $\mathrm{ScN}$ system. Red squares represent mean-field Curie temperatures $T_{c}^{\mathrm{MF}}$, and blue squares represent cluster variation method Curie temperature $T_{c}^{\mathrm{CVM}}$.

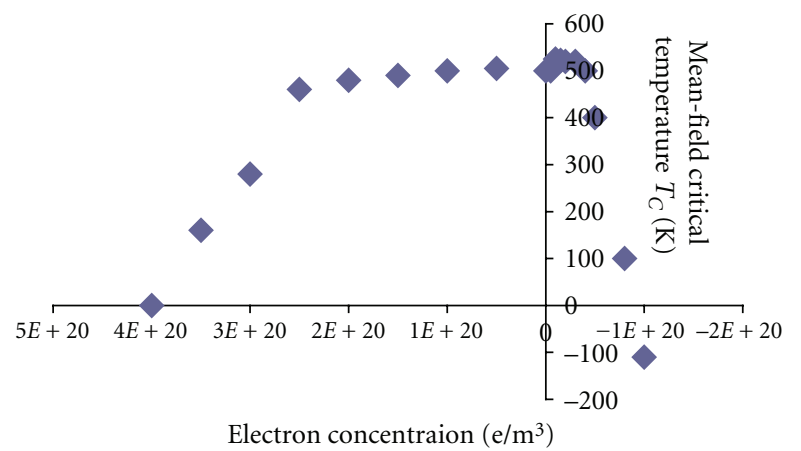

Figure 4: The mean-field critical Curie temperature $T_{c}$ as a function of electron concentration of Mn-doped ScN system.

are rather typical for Mn-doped ScN samples. At least one sample with $3 \% \mathrm{Mn}$ was reported to show a ferromagnetic behavior up to $50 \mathrm{~K}$ [18]. The $n$-type doping in $\mathrm{ScN}$ is generated from nitrogen vacancies. Consequently, we carried out some calculations with about $10 \% \mathrm{Mn}$ and $3 \%$ nitrogen vacancy $\mathrm{V}_{\mathrm{N}}$ corresponding to two $\mathrm{V}_{\mathrm{N}}$ per cell. The $3 \% \mathrm{~V}_{\mathrm{N}}$ corresponds to doping by about $6 \times 10^{19} \mathrm{e} / \mathrm{cm}^{3}$. We found that $T_{c}^{\mathrm{MF}}$ drops by approximately $11 \%$.

\section{Conclusions}

In summary, our direct supercell simulations show that Mndoped $\mathrm{ScN}$ is promising DMS systems with extraordinary optical, structural, and magnetic properties. By using total energy calculations, we found that global energy minimum of $\mathrm{MnN}$ is obtained for $\mathrm{ZB}$ structure. As the compound is slightly compressed, the energy minimum corresponds 
to the RS structure exhibiting a magnetic moment of a value of $2.52 \mu_{\mathrm{B}}$ in disagreement with the experimental distorted RS $\theta$-phase found for Mn. Our calculations for the magnetic exchange interactions indicate the possibility of above room temperature $T_{c}$ for Mn concentrations of about $10 \%$. Our results on the effects of doping indicate that room temperature ferromagnetism is achieved by maintaining the $n$-type carrier concentration below $10^{20} \mathrm{~cm}^{-3}$. Thus, the Mn-doped ScN DMS is expected to be the key candidate component for life time spintronic devices. In addition, we found that $\mathrm{Mn}_{\mathrm{x}} \mathrm{Sc}_{1-\mathrm{x}} \mathrm{N}$ alloys undergo an isostructural phase transition from $\mathrm{MnN} \theta$-phase to $h$-ScN at a critical hydrostatic pressure of $18 \mathrm{GPa}$. Such a transition could be associated with several anomalous properties.

\section{Acknowledgments}

The author acknowledges E. Sigmund and his research team at Brandenburg Technical University in (BTU), CottbusGermany, for useful discussions. Computational support has been provided by the Theoretical physics institute at BTU in Germany.

\section{References}

[1] A. Herwadkar and W. R. L. Lambrecht, "Mn-doped ScN: a dilute ferromagnetic semiconductor with local exchange coupling," Physical Review B, vol. 72, no. 23, Article ID 235207, 6 pages, 2005.

[2] H. A. Al-Brithen, H. Yang, and A. R. Smith, "Incorporation of manganese into semiconducting $\mathrm{ScN}$ using radio frequency molecular beam epitaxy," Journal of Applied Physics, vol. 96, no. 7, pp. 3787-3792, 2004.

[3] J. L. Xu, M. van Schilfgaarde, and G. D. Samolyuk, "Role of disorder in Mn:GaAs, Cr:GaAs, and Cr:GaN," Physical Review Letters, vol. 94, no. 9, Article ID 097201, 4 pages, 2005.

[4] L. Bergqvist, O. Eriksson, J. Kudrnovsky, V. Drchal, P. Korzhavyi, and I. Turek, "Magnetic percolation in diluted magnetic semiconductors," Physical Review Letters, vol. 93, no. 13, Article ID 137202, 4 pages, 2004.

[5] I. Zutic, J. Fabian, and S. D. Sarma, "Spintronics: fundamentals and applications," Reviews of Modern Physics, vol. 76, no. 2, pp. 323-410, 2004.

[6] P. Dismukes, W. M. Yim, and V. S. Ban, "Epitaxial growth and properties of semiconducting ScN," Journal of Crystal Growth, vol. $13-14$, pp. 365-370, 1972.

[7] N. Farrer and L. Bellaiche, "Properties of hexagonal ScN versus wurtzite GaN and InN," Physical Review B, vol. 66, no. 20, Article ID 201203, 4 pages, 2002.

[8] A. Leineweber, R. Niewa, H. Jacobs, and W. Kockelmann, "The manganese nitrides $\eta-\mathrm{Mn}_{3} \mathrm{~N}_{2}$ and $\theta-\mathrm{Mn}_{6} \mathrm{~N}_{5+x}$ : nuclear and magnetic structures," Journal of Materials Chemistry, vol. 10, no. 12, pp. 2827-2834, 2000.

[9] P. Hohenberg and W. Kohn, "Inhomogeneous electron gas," Physical Review, vol. 136, no. 3B, pp. B864-B871, 1964.

[10] W. Kohn and L. J. Sham, "Self-consistent equations including exchange and correlation effects," Physical Review, vol. 140, no. 4A, pp. A1133-A1138, 1965.

[11] A. D. Becke, "Density-functional exchange-energy approximation with correct asymptotic behavior," Physical Review A, vol. 38, no. 6, pp. 3098-3100, 1988.
[12] J. P. Perdew, K. Burke, and Y. Wang, "Generalized gradient approximation for the exchange-correlation hole of a manyelectron system," Physical Review B, vol. 54, no. 23, pp. 1653316539, 1996.

[13] P. Blaha, K. Schwarz, G. K. H Madsen, D. Kvasnicka, and J. Luitz, Crystal Properties, Technische Universität, Wien, Austria, 2001.

[14] P. Blaha, K. Schwarz, and J. Luitz, WIEN97: A Full Potential Linearized Augmented Plane Wave Package for Calculating Crystal Properties, Karlheinz Schwarz, Technische Universität, Wien, Austria, 1999.

[15] V. Ranjan, S. Bin-Omran, L. Bellaiche, and A. Alsaad, "Isostructural phase transitions in $\mathrm{GaN} / \mathrm{ScN}$ and $\mathrm{InN} / \mathrm{ScN}$ superlattices," Physical Review B, vol. 71, no. 19, Article ID 195302, 6 pages, 2005.

[16] M. A. Ruderman and C. Kittel, "Indirect exchange coupling of nuclear magnetic moments by conduction electrons," Physical Review, vol. 96, no. 1, pp. 99-102, 1954.

[17] T. Kasuya, "A Theory of Metallic Ferro- and Antiferromagnetism on Zener's Model," Program on Number Theory and Physics, vol. 16, p. 45, 1956.

[18] C. Constantin, "Growth, Structural, Electronic and Optical Characterization of Nitride Semiconductors Grown by rfPlasma Molecular Beam Epitaxy," Ph.D. thesis, Ohio University, Athens, Ohio, USA, 2005. 

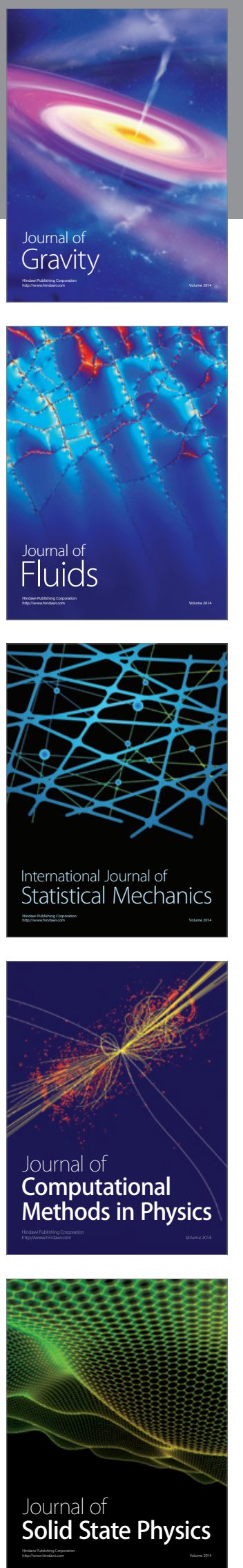

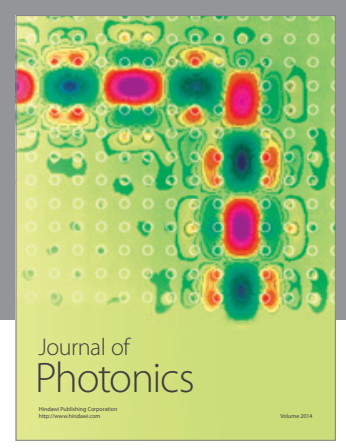

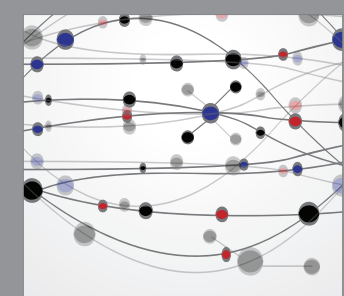

The Scientific World Journal
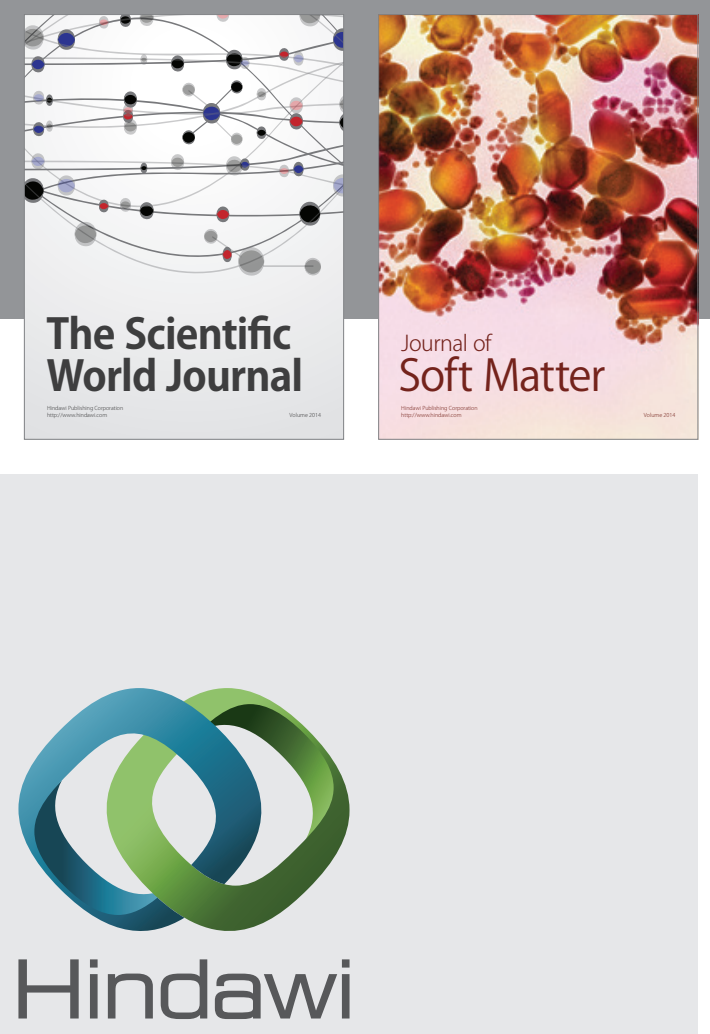

Submit your manuscripts at

http://www.hindawi.com
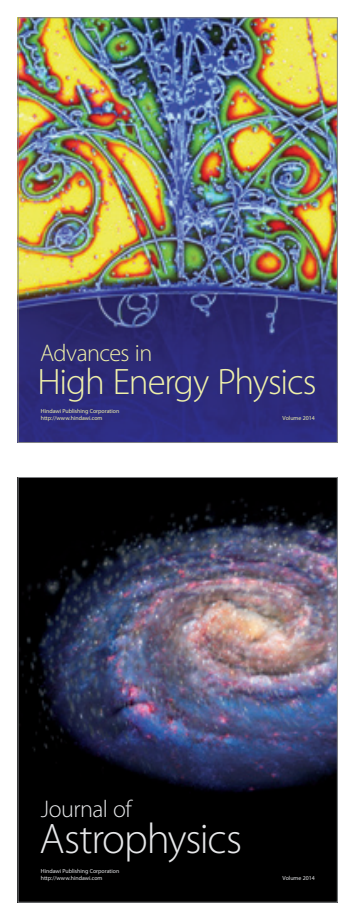
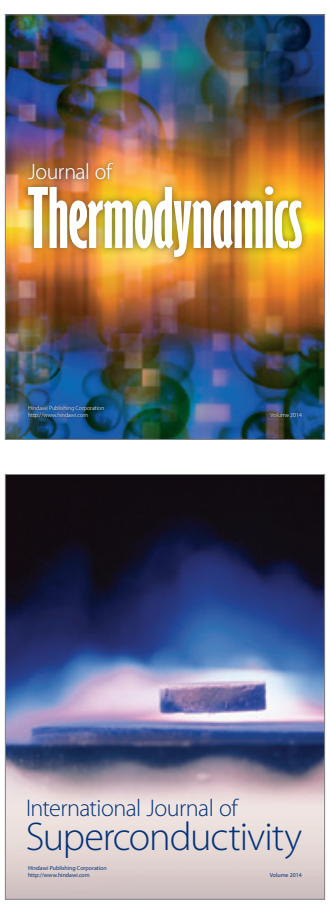
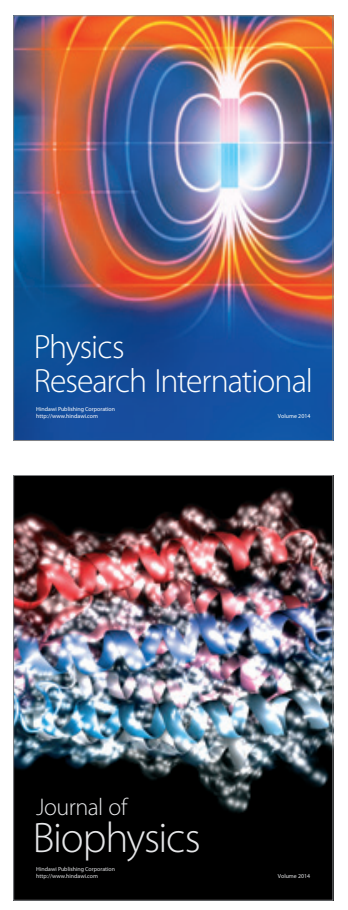
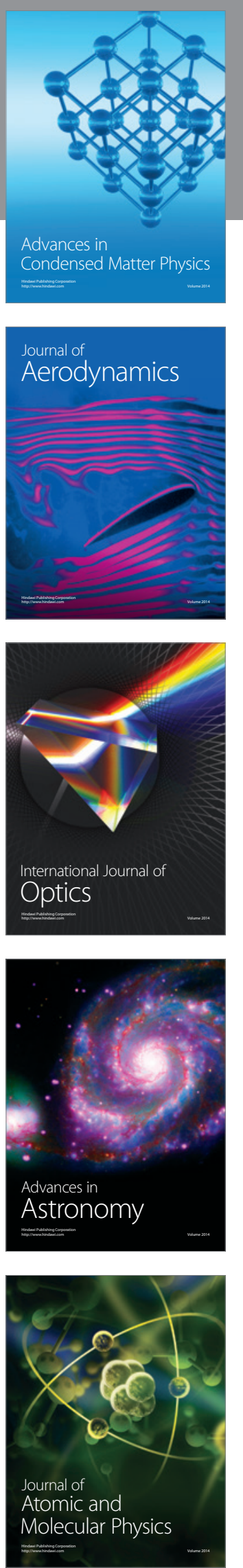